\title{
The Chroma Software System for Lattice QCD
}

\author{
Robert G. Edwards ${ }^{\mathrm{a}}$ SciDAC and LHPC Collaboration, Bálint Joób UKQCD Collaboration \\ ${ }^{a}$ Thomas Jefferson National Accelerator Facility, Newport News, VA 23606, USA \\ bSchool of Physics, University of Edinburgh, Edinburgh, Scotland, UK, EH9 3JZ
}

We describe aspects of the Chroma software system for lattice QCD calculations. Chroma is an open source $\mathrm{C}++$ based software system developed using the software infrastructure of the US SciDAC initiative. Chroma interfaces with output from the BAGEL assembly generator for optimised lattice fermion kernels on some architectures. It can be run on workstations, clusters and the QCDOC supercomputer.

\section{INTRODUCTION}

We present the Chroma software system 1] for lattice QCD (LQCD) calculations. Chroma aims to provide a computational LQCD toolbox which is flexible, portable and efficient on a wide range of architectures from desktop workstations to clusters, commercial machines and new architectures such as the QCDOC 2]

Development on Chroma started at the $\mathrm{JLab}^{1}$ for the U.S. lattice community, in particular the LHPC collaboration [3, using software from the U.S. SciDAC initiative 4. This effort has been joined by the UKQCD collaboration [5] who have been contributing to the effort on all levels.

To achieve the goals of flexibility, portability and efficiency, Chroma relies on several layers of SciDAC and UKQCD software.

\subsection{SciDAC Software Hierarchy}

Toward the end of 2000, the U.S. Lattice community embarked on an ambitious project through the U.S. SciDAC initiative to standardise a set of software components in order to allow the effective exploitation of computing resources for LQCD. The following levels of software infrastructure were defined:

QCD Message Passing (QMP): provides a message passing API tailored to LQCD calculations. QMP was designed to take advantage of the specialised communication hardware of emerging architectures, such as

${ }_{1}$ in full: Thomas Jefferson National Accelerator Facility the Serial Communications Unit (SCU) of the QCDOC, or the capabilities of Myrinet Network devices.

Level 1 - QCD Linear Algebra (QLA): defines operations to be performed at each site of the lattice. Primitives include $S U(3)$ matrix-matrix and matrix-colour vector multiplications.

Level 2 - QCD Data Parallel (QDP): provides lattice-wide operations such as basic linear algebra for lattice-wide fields.

Level 3 - Special optimised software: will provide portable interfaces to highly optimised and machine-dependent pieces of code such as assembly-coded Dslash operators.

A recent addition to this hierarchy is the $\mathbf{Q C D}$ I/O (QIO) sublayer which provides a record oriented I/O API. XML metadata and binary data can be packaged as separate records of a single file for transmission to future Web/Grid services.

Level 3 of the SciDAC hierarchy is not yet as mature as the other levels, hence Chroma uses optimised Dslash operators from a variety of sources, such as the Pentium 4 SSE assembler code developed at the JLab [6], or the output of the BAGEL [7] generator.

\section{2. $\mathrm{QDP}++$}

$\mathrm{QDP}++$ is a $\mathrm{C}++$ implementation of the QDP level of the SciDAC hierarchy, which Chroma 
uses as its foundation. QDP ++ defines latticewide types and allows lattice-wide expressions. It has been integrated with the QIO framework and with modules to provide XML based I/O (e.g. for parameter reading, or processing QCDML markup [8]).

\subsection{Lattice-Wide Types}

$\mathrm{QDP}++$ models the tensor product structure of LQCD objects through a series of nested templates. E.g: the indices of lattice fermion fields can follow the structure:

sites $\otimes$ spins $\otimes$ colours $\otimes$ complex numbers

$\mathrm{QDP}++$ would model this through the following $\mathrm{C}++$ templated type:

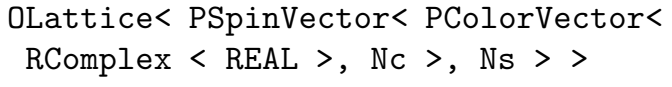

where REAL is the type of the complex components, defined as either float or double. Nc and $\mathrm{Ns}$ are the numbers of spin and colour components defined during configuration.

$\mathrm{QDP}++$ operates on such types by recursing down the tower of templates. To multiply the above fermion type by a scalar, one would first loop through the indices of the OLattice type and for each one, call the multiply operation for the PSpinVector type and so on.

The order of the templates is not fixed in principle and could be permuted in order to take advantage of different parallel architectures. The templated types are aliased to fixed type names such as LatticeFermion that are used by higherlevel codes.

\subsection{Template Expressions, PETE}

$\mathrm{QDP}++$ provides lattice-wide arithmetic expressions by overloading operators. The AXPY operation

$z \leftarrow \alpha x+y$

for lattice fermion fields $x, y, z$ and scalar $\alpha$ is coded as:

$\mathrm{z}=\mathrm{a} * \mathrm{x}+\mathrm{y}$.

To eliminate temporaries in expressions, $\mathrm{QDP}++$ uses the Portable Expression Template
Engine $(\mathrm{PETE})^{2}$ 9. The $\mathrm{C}++$ binary operators are redefined to transform the expression into an expression template type. On assignment, storage for the result and the expression template, which includes references to the operands, are all given as arguments to an evaluate() function. This is done at compile time through the template instantiation mechanism. The process for the AXPY operation of Eq. (II) is shown in Fig. [1]

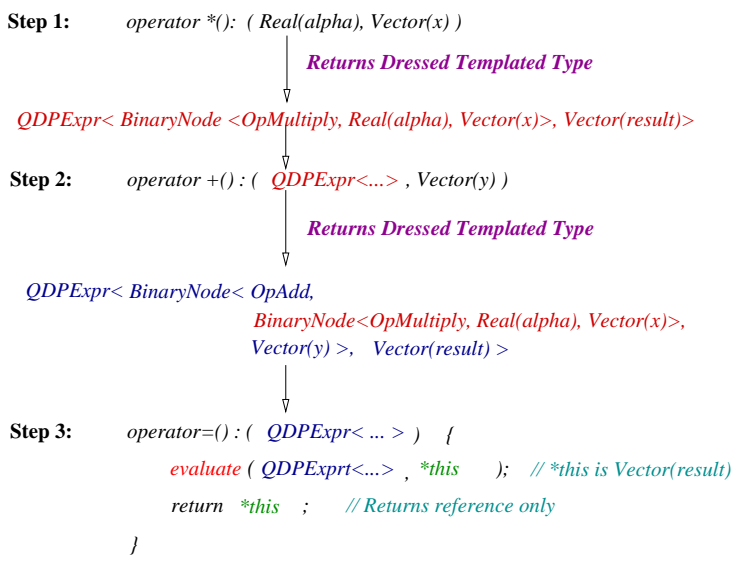

Figure 1. Expression Transformation

Templated evaluate() functions can be specialised for specific expression templates, and the specialised functions can call optimised subroutines, e.g. fast AXPY like functions.

It may be possible to optimise general expressions even further by applying PETE techniques at all levels of our template hierarchy rather than just at the OLattice level as is done now.

\section{CHROMA}

Chroma developed to serve the needs of the LHPC and UKQCD collaborations, which have included spectroscopy, decay constant, nucleon form factor and structure function moment calculations. The authors are also interested in investigating chiral fermion actions. Hence the code contains Wilson, Domain Wall and Overlap fermion operators and numerous inverters.

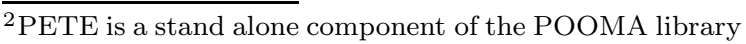


Code also exists to compute hadronic 2-point and 3-point correlation functions. Furthermore, UKQCD researchers have written an $A S q T A D$ fermion operator in the staggered library.

\subsection{Chroma and Efficiency}

By optimising QDP ++ as discussed previously and using high performance kernels in our linear operators we have achieved high performance on workstations, clusters and the QCDOC.

Fig. 2 shows single-precision performance measurements with an even-odd preconditioned Wilson Dirac operator on 4 nodes of a QCDOC using the assembler Dslash from BAGEL. We show the performance of the Dslash, the Dirac operator, a naively implemented Conjugate Gradients (CG) solver and an optimised CG solver, which makes direct calls to the Dslash routine and fuses the application of the Dirac Matrix with the computation of the norm of the result where possible.

The Dslash routine (from BAGEL) was designed to amortise communications latency at a local lattice size of 256 sites. Scaling from this point to larger volumes can be seen in Fig. 2. The cost of the additional vector operations needed to combine the Dslash applications into the Dirac operator appears as the difference between the red and black bars. The difference between the general CG and the optimised one is about $2-3 \%$ of peak over the whole range of volumes, showing that the overhead from $\mathrm{QDP}++$ expressions is very small.

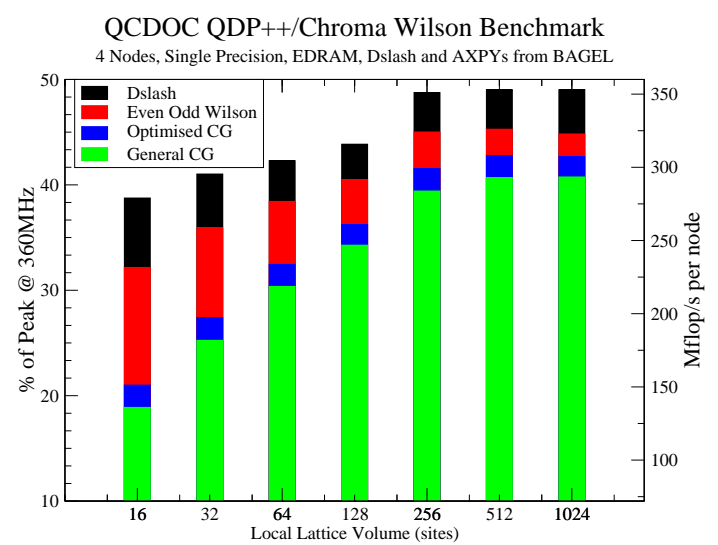

Figure 2. Performance on the QCDOC

\section{CONCLUSIONS}

We have discussed the SciDAC software hierarchy and elements of the QDP ++ and Chroma software suites for lattice QCD and have shown how the latter may be optimised in order to achieve high efficiency through template expressions and by interfacing cleanly with third party high performance libraries.

Due to lack of space we have had to gloss over several other nice features of Chroma and $\mathrm{QDP}++$ such as the many applications already existing in the code base and our build system which uses the GNU Autotools, allowing for easy configuration and building. These details are deferred to a forthcoming publication.

Our future plans include the implementation of gauge configuration generating algorithms for quenched and dynamical fermions. At the time of writing, the basic class framework for this is already in place.

QMP, QDP ++ and Chroma are open source software and are freely available through anonymous CVS, details of which can be found in [1].

\section{ACKNOWLEDGEMENTS}

R. G. Edwards is supported under DOE contract DE-AC05-84ER40150 under which the Southeastern Universities Research Association (SURA) operates the Thomas Jefferson National Accelerator Facility. B. Joó gratefully acknowledges funding under PPARC Grant PPA/G/0/2002/00465. We thank P. A. Boyle for assistance with the BAGEL assembly generator.

\section{REFERENCES}

1. http://www.jlab.org/ edwards/chroma

2. P. A. Boyle, these proceedings

3. http://www.jlab.org/ dgr/lhpc

4. http://www.lqcd.org/scidac

5. http://www.ph.ed.ac.uk/ukqcd

6. C. McClendon, JLAB-THY-01-29, http://www.jlab.org/ ^edwards/qcdapi/reports/dslash_p4.pd

7. P. A. Boyle, in preparation

8. http://www. lqcd.org/ildg

9. http://www.codesourcery.com/pooma 